\title{
La notion de famille repensée. Portrait succinct de quelques familles lesbiennes de la région de Québec
}

\author{
Ann Robinson et Madeleine Côté
}

How can lesbians cope with social constraints, discrimination, marginalisation and sometimes violence related to their life choices (marital life and parenthood)? Research was conducted among lesbian families living in Quebec City and Winnipeg. The authors indicate that the conclusions were the same in both environments and gave way to broader definitions of the concept of family. The text is a brief summary of the results for the Quebec City region.

On définit traditionnellement la famille comme « un regroupement de personnes apparentées vivant sous le même toit ${ }^{1} »$, comme le père, la mère et leurs enfants. Mais cette institution a évolué de telle sorte que, à la fois dans les écrits scientifiques et dans les écrits populaires, il est de plus en plus question d'autres formes de famille, par exemple la famille monoparentale. ${ }^{2}$ En partant de la prémisse que les lesbiennes sont des femmes « ordinaires », qui vivent des relations amoureuses, et comme toutes les femmes du monde, "tombent en amour », ont des peines d'amour, se relèvent et continuent à avancer, peuvent mettre au monde des enfants, vivre avec eux et elles, parfois seule, parfois avec une conjointe, pourquoi alors ne pas reconnaître ces cellules comme des familles et les nommer sans hésitation « familles lesbiennes ${ }^{3}$ ? . Dans le cadre d'une recherche subventionnée par Condition Féminine Canada durant l'année 1997, deux équipes de recherche, l'une à Winnipeg ${ }^{4}$ et l'autre à Québec ${ }^{5}$, ont tenté de cerner comment des lesbiennes conjointes ou mères composent avec les contraintes sociétales, la discrimination, la marginalisation et parfois la violence, quant à leur conjugalité, à leur parentalité et aux avantages sociaux ${ }^{6}$. Le présent texte, très succinct, se limite aux résultats du volet québécois de l'enquête.

Cette recherche s'inscrivait dans un cadre socio-juridique. Pour atteindre les objectifs du cadre juridique, deux membres de l'équipe ont procédé au repérage, à l'analyse et à la critique d'une cinquantaine de causes de 
jurisprudence portant de près ou de loin sur l'homosexualité, le lesbianisme, les lesbiennes ou les homosexuels. ${ }^{7}$ Parallèlement, les autres membres des deux équipes ont cherché à cerner les expériences et les perceptions de lesbiennes quant à leur conjugalité, à leur parentalité et aux avantages sociaux liés à leur situation de famille. Pour ce faire, nous avons mené quinze entrevues dans la région de Québec, avec quatorze lesbiennes ${ }^{8}$ d'âges variés qui ont vécu ou vivent des expériences discriminantes dans les trois domaines désignés. Faute d'espace, la méthodologie adoptée, conforme aux standards des disciplines en cause, ne sera pas développée dans le présent texte. Nous nous contenterons de brosser un tableau de ce que les conclusions permettent d'apercevoir.

Notre recherche s'inscrivant dans une perspective féministe conscientisante, deux membres de l'équipe de Québec, lesbiennes, ont choisi d'être aussi répondantes. En effet, il nous est apparu fondamental de nous impliquer dans la recherche que nous menions avec des lesbiennes sujets de recherche.

\section{Les répondantes : Qui sont-elles?}

Les répondantes avaient en moyenne 43,1 ans lors de l'entrevue. Deux $d^{\prime}$ 'entre elles vivent et travaillent en milieu rural, à plus d'une centaine de kilomètres de Québec, onze autres vivent dans la ville de Québec, dans la banlieue immédiate ou dans les environs, et travaillent à Québec; la dernière vit et travaille en banlieue. Toutes ont un emploi rémunéré sauf une qui était retournée aux études au moment de l'entrevue. Elles exercent des fonctions de niveau professionnel ou technique, ou encore de soutien dans le domaine de l'éducation, ou sont intervenantes dans le domaine de la santé traditionnelle ou alternative ou professeures, l'une est contractuelle en loisirs, une est cheffe d'entreprise, une est avocate et une dernière est animatrice en pastorale. Elles sont plus scolarisées que la moyenne des Canadiennes qui se situe autour des études secondaires. En effet, dix répondantes ont achevé des études universitaires, alors que trois autres ont terminé des études collégiales, ou obtenu l'équivalent. Enfin, la répondante qui possède un diplôme d'études secondaires a fait pour sa part des études en médecine alternative en dehors du réseau officiel québécois de l'éducation.

Ces répondantes ont majoritairement des enfants autour d'elles. Douze enfants se partagent six mères lesbiennes, douze vivent dans l'entourage de six autres lesbiennes. Un total de huit répondantes sur quatorze parta- 
gent leur vie avec des enfants puisque certaines mères lesbiennes vivent ou ont une relation amoureuse avec une autre mère lesbienne, alors que six répondantes n'ont aucun enfant directement dans leur vie. Mais parmi ces dernières, deux jeunes lesbiennes souhaitent avoir éventuellement, par insémination artificielle ou adoption, un ou des enfants alors qu'une dernière s'occupe de la fille d'une amie.

La situation de famille des répondantes s'est avérée très variée. Sontelles célibataires, conjointes de fait, «mariées », cheffes de famille monoparentale ou mères célibataires? Au moment de l'entrevue, elles se décrivent soit comme célibataires avec amante, mariées à une femme, conjointes de fait vivant ou non avec une conjointe, ou même célibataires ayant une amante. Quant à leur situation de famille antérieure, elles étaient, célibataires lesbiennes ou hétérosexuelles, mariées à un homme ou conjointes de fait vivant avec une autre femme sous le même toit.

Ces données nous permettent de constater que « ces femmes qui aiment une femme », ont visiblement de la difficulté à nommer leur réalité, et que le passage au lesbianisme prend parfois des détours et même des chemins tortueux. Par ailleurs, plus du tiers des répondantes nous assurent qu'elles ont toujours été lesbiennes, en ce sens qu'elles n'ont eu ou désiré que des relations amoureuses avec des femmes.

\section{Les répondantes : Comment se nomment-elles?}

À la question sur l'orientation sexuelle, huit répondantes affirment sans hésitation qu'elles sont lesbiennes et six éprouvent une certaine difficulté à se dire lesbiennes. Parmi ces dernières, deux disent détester les catégories, et trois autres préfèrent se qualifier de gaies plutôt que de lesbiennes. Enfin, une dernière n'arrive tout simplement pas à se nommer. ${ }^{9}$ On peut aisément expliquer leur difficulté à utiliser le mot lesbienne, souvent connoté négativement par leur entourage et parfois chargé de mépris, haine, colère, violence et même ironie. Il n'est donc pas surprenant que six répondantes soient incapables d'utiliser le mot lesbienne quand elles parlent d'elles, alors qu'elles vivent une relation amoureuse avec une femme. Pour les huit autres répondantes qui se disent spontanément lesbiennes, leur affirmation correspond à un geste d'acceptation, voire à un engagement politique.

Les répondantes disent être des femmes ordinaires, qui « tombent en amour ", vivent des relations amoureuses avec les hauts et les bas que cela entraîne. Elles se présentent comme des femmes compréhensives, attentionnées envers leur conjointe, empathiques, compatissantes et parfois jalouses. 
Elles investissent beaucoup dans leurs relations amoureuses, veulent qu'elles durent le plus longtemps possible et sont prêtes à sacrifier un peu de leur liberté ou de leur indépendance pour obtenir la reconnaissance de la réalité de leur couple, de leur famille lesbienne. Dix répondantes ont parlé de leur conjugalité lesbienne, de la marginalisation et de la discrimination dont elles sont l'objet, et de la façon dont elles désirent voir leur réalité conjugale considérée par la loi. Leurs propos nous apparaissent toujours pertinents même si les gouvernements fédéral et québécois ont par la suite adopté des lois qui reconnaissent en partie la conjugalité de fait lesbienne et homosexuelle. ${ }^{10}$ En effet, ces deux lois n'ont aucun effet sur le Code civil qui s'avère être la seule loi en vigueur au Québec lorsqu'il est question de la famille et des relations entre les divers membres de la famille, comme le mariage, la conjugalité de fait, la filiation ou les successions.

L'analyse des données fait ressortir que l'ensemble des répondantes refuse le maintien de la situation actuelle de non-existence légale qui, selon elles, entraîne nécessairement la marginalisation et l'ostracisme des familles lesbiennes. Optant parfois pour plus d'une hypothèse, elles expriment qu'elles devraient avoir le choix de faire reconnaître légalement leur conjugalité lesbienne. Elles ont également insisté sur la garantie de protection que devrait leur assurer la loi mettant ainsi en échec toute possibilité d'interprétation malveillante de la part des tribunaux. Trois répondantes opteraient pour le mariage légal tel qu'il est actuellement décrit au Code civil du Québec. Huit répondantes insistent pour que ce même Code civil reconnaisse et réglemente l'union de fait lesbienne. Six lesbiennes seraient satisfaites si le Code leur permettait de signer un contrat notarié d'union de fait, à la condition d'avoir une garantie que ce contrat soit reconnu par les tribunaux. Enfin, trois répondantes ont spécifié qu'elles préféreraient que la situation actuelle se règle en permettant aux lesbiennes de signer une déclaration volontaire de conjugalité dans un registre central, sorte de $\mathrm{PaCS}^{11}$ version québécoise.

Nos répondantes ont finalement formulé leur propre définition de la famille, de la conjugalité de fait et des relations lesbiennes. Pour la plupart, deux personnes qui vivent une relation de couple et qui décident de « faire vie commune ", constituent une famille. Et si ces deux personnes sont des femmes, ce sera une famille lesbienne. En compilant toutes les définitions proposées, nous avons constaté à quel point nos répondantes espèrent que la société évolue rapidement de façon à ce qu'elles puissent vivre ouvertement et au grand jour leur relation de famille et de conjugalité. Elles 
considèrent souvent leurs amies et amis, surtout lesbiennes et gais, comme membres à part entière de leur famille. Elles intègrent tout naturellement leur conjointe ou amante à leur famille, de même que leurs enfants ou les enfants de leur conjointe. La plupart d'entre elles d'ailleurs n'ont aucune difficulté à affirmer qu'elles forment une famille avec leur conjointe. Quelques-unes éprouvent cependant de la difficulté à considérer la notion de famille sans la présence d'enfant. Par ailleurs, aucune d'elles n'a identifié la communauté lesbienne et gaie de sa région comme faisant partie intégrante de sa famille.

\section{La parentalité lesbienne}

Trois répondantes ont accepté de nous parler de leur réalité de mères. Nous leur avons spécifiquement demandé de nous faire part de leurs récits de vie de mères lesbiennes et des formes de violence ou de discrimination qu'elles ont vécues. Une bonne part de notre rapport final a été consacré à l'analyse transactionnelle de leurs discours. Nous constatons de façon générale qu'elles sont en total désaccord avec le fait qu'une femme ne peut être à la fois mère et lesbienne. Bien au contraire, une répondante pense qu'être mère lesbienne la rend plus tolérante et conciliante, à la fois avec ses enfants, ses étudiantes et étudiants et les amies et amis de ses enfants. Pour une autre, l'orientation sexuelle est tout à fait secondaire en regard de sa réalité de mère. Une dernière considère enfin qu'avoir une mère lesbienne pour un enfant n'est certainement pas mieux ni pire que d'avoir une mère très prise par sa vie professionnelle, artiste, obèse, alcoolique, toxicomane, sportive ou célèbre.

Nous entendons parfois certaines personnes s'insurger contre le fait que des lesbiennes puissent avoir ou conserver la garde de leurs enfants. Conséquemment, des juges ont accordé cette garde au père à seule fin que ces enfants soient élevés dans un milieu hétérosexuel, ${ }_{1}^{12}$ pour éviter qu'ils ou elles deviennent plus tard lesbiennes ou homosexuels. Pourtant les recherches menées aux États-Unis et en Europe sur cette question sont concluantes : il n'existe aucun lien entre l'orientation sexuelle des parents et celle de leurs enfants. ${ }^{13}$ Nous avons demandé aux répondantes ce qu'elles-mêmes pensaient de cette opinion assez répandue dans la classe juridique. Toutes considèrent que cela relève d'un préjugé défavorable à l'égard des lesbiennes, d'une manifestation de lesbophobie caractéristique d'une société hétéropatriarcale. Au contraire, elles ont la conviction que leurs enfants jouiront d'une plus grande ouverture d'esprit, de plus de 
tolérance face à toutes les formes de marginalité, du fait d'avoir vécu avec une mère lesbienne. Selon elle, leurs enfants étant régulièrement imprégnés de deux modèles d'expression de la sexualité humaine, leurs enfants seront plus à même de faire un choix éclairé lorsque viendra le temps de reconnaître leur propre orientation sexuelle. ${ }^{14}$

Enfin, nous avons demandé à onze répondantes comment les systèmes législatifs et judiciaires canadiens et québécois pourraient et devraient reconnaître et protéger la parentalité lesbienne. Leurs commentaires sont toujours pertinents, même après l'adoption de la loi fédérale C-23 et de la Loi 32 du Québec. ${ }^{15}$ Toutes les répondantes réclament une reconnaissance légale de la parentalité lesbienne qu'elle soit biologique ou adoptive. En effet, alors que certaines répondantes voudraient avoir accès à l'insémination artificielle dans le cadre d'un projet de conjugalité, d'autres aimeraient adopter en couple un ou des enfants, ou adopter les enfants de leur conjointe. Dans un deuxième temps, certaines vont plus loin en affirmant qu'il serait important d'agir de manière à ce que les mentalités de la population et des représentantes et représentants des systèmes législatifs et judiciaires évoluent, et pour cela elles croient que des campagnes de sensibilisation pourraient être utiles pour démontrer que les lesbiennes qui le désirent peuvent être des mères comme les autres.

\section{Droit à la différence ou indifférence?}

En acceptant de parler, de répondre aux questions lors des entrevues, ces lesbiennes auront réussi à témoigner de l'existence des familles lesbiennes québécoises et à en tracer un portrait éloquent. Les récits sur la discrimination et la violence vécues par ces familles tant de la part de la société que de la part des membres de leur famille élargie et ceux de celles vécues par leurs enfants, de même que la discrimination dans les milieux de travail ${ }^{16}$ et les milieux sociaux ${ }^{17} \mathrm{n}^{\prime}$ ont pas été repris ici. Cependant, ils ont été en partie évoqués dans des textes déjà publiés et nous gardons l'espoir de voir cette recherche publiée dans son intégralité.

Droit à la différence ou droit à l'indifférence ? Que veulent les lesbiennes ? Celles que nous avons rencontrées sont certes différentes des femmes hétérosexuelles. Mais elles ne le sont pas plus que nous le sommes de nos voisines, même si ces dernières sont lesbiennes; que les lesbiennes jeunes sont différentes des lesbiennes âgées, etc. Chaque personne est unique et les différentes chartes des droits reconnaissent l'égalité entre les personnes. Et pourtant, les lesbiennes sont encore discriminées directement ou ouvertement 
dans quelques lois canadiennes et québécoises. ${ }^{18} \mathrm{Il}$ faut donc continuer à combattre ces lois qui nous identifient comme des citoyennes de second ordre, des sous-femmes, car cette situation ne peut qu'entraîner la marginalisation et l'ostracisme de l'ensemble de la classe lesbienne et par voie de conséquence, de toutes les familles lesbiennes.

\section{Notes}

${ }^{1}$ Le nouveau Petit Robert (éd. 1993), p. 890.

2 À titre d'exemple de cette évolution, il suffit de consulter le rapport de recherche soumis à la ministre de l'Emploi et de la Solidarité et au garde des Sceaux, ministre de la Justice de la France en juin 1998. Irène THÉRY, Couple, Filiation et Parenté aujourd'hui, Le droit face aux mutations de la famille et de la vie privée (Paris : Éditions Odile Jacob, 1998).

${ }^{3}$ En France, depuis quelques années, on identifie ces familles comme des familles « homoparentales » Martine GROSS (dir.), Homoparentalités, état des lieux, Parenté et différence des sexes (Paris : ESF éditeur, 2000).

${ }^{4}$ L'équipe de Winnipeg, dirigée par la docteure Sandra Kirby, professeure de sociologie à l'Université de Winnipeg, comprenait également Heidi Streu et Susan Riley, assistantes de recherche.

5 À Québec, l'équipe était dirigée par Ann Robinson, professeure de droit à l'Université Laval, laquelle était assistée de Madeleine Côté et Johanne Carrier, assistantes de recherche.

${ }^{6}$ Chacune des deux équipes a produit un rapport, tel que le stipulait le devis de recherche. Pour le Manitoba, voir Sandra KIRBY, Manitoba Struggle for Rights : Lesbians and their Families, 31 mars 1997, document inédit, 130 pages; et pour le Québec, voir Ann Robinson et Madeleine Côté, La famille lesbienne au Québec : Une réalité méconnue et marginalisée (31 mars 1997), document inédit, 98 pages. Ces documents sont malheureusement restés inédits, Condition Féminine Canada ayant refusé d'investir des fonds pour les publier.

${ }^{7}$ Cette partie de la recherche a fait l'objet d'un rapport distinct. Voir Susan P. Riley, Lesbian Struggles for Human Rights, The Legal Story (janvier 1997), document inédit, 30 pages.

${ }^{8}$ L'expérience de vie d'une des répondantes justifiait de lui proposer deux entrevues, l'une portant sur la conjugalité, l'autre sur la parentalité.

${ }^{9}$ Ces données correspondent aux données recueillies par Line Chamberland auprès de lesbiennes de Montréal. Voir Mémoires lesbiennes (Montréal : Les éditions du Remue-ménage, 1996) chapitre 8 : Les pratiques identitaires, p. 179-222. 
${ }^{10}$ Loi C-23, Loi visant à moderniser le régime d'avantages et d'obligations dans les Lois du Canada; Loi 32, Loi modifiant diverses dispositions législatives concernant les conjoints de fait.

${ }^{11}$ Cette nouvelle réalité juridique vient de France. La loi autorisant les PaCS a été adopté à l'automne 1999. « Un pacte civil de solidarité est un contrat conclu par deux personnes physiques majeures, de sexe différent ou de même sexe, pour organiser leur vie commune ». Alia AOUN, Le PaCS, Delmas express (Paris : 2000) 5.

${ }^{12}$ Ann Robinson, « Lesbiennes, conjointes et mères : les exclues du droit civil québécois ". Des droits à reconnaître, les lesbiennes face à la discrimination. Montréal : Les Éditions du remue-ménage, 1998. 21-68.

${ }^{13}$ Danielle Julien, Monique Dubé et Isabelle Gagnon, « Le développement des enfants de parents homosexuels comparé à celui des enfants de parents hétérosexuels ", Revue québécoise de psychologie 15.3 (1994); Geneviève Delaisi de Parseval, « La construction de la parentalité dans les couples de même sexe » dans Daniel Borrillo, Éric Fassin et Marcela Iacub, Au-delà du PaCS, L'expertise familiale à l'épreuve de l'homosexualité (Paris : PUF, 1999) 225-44.

${ }^{14}$ Les chercheuses et chercheurs qui ont travaillé sur cette question, sont arrivés aux mêmes résultats. Voir Ibid.

15 Supra, note 11.

${ }^{16}$ Cependant, cette partie de la recherche a déjà fait l'objet d'une publication pour ce qui est des données québécoises recueillies. Voir Ann Robinson, « Du sexisme à l'hétérosexisme et à la lesbophobie " dans $D^{\prime}$ autres violences à dire (Actes du colloque tenu à Trois-Rivières le 13 mai 1997 dans le cadre du 65 $5^{\circ}$ congrès de l'ACFAS) sous la direction de Michèle Clément, Sylvie Gravel et Dominique Damant, CRI-VIFF, collection RÉFLEXIONS n8, Université Laval, Décembre 1998, p.87-110.

17 Il ne faut pas oublier également que dans le cadre de cette recherche, 15 entrevues ont été menées dans la région de Winnipeg. Les résultats de ces entrevues ressemblent en tous points à ceux obtenus au Québec. Malheureusement nous n'avons jamais pu trouver les fonds nécessaires à la publication d'un rapport synthèse comparatif entre le Québec et le Manitoba.

${ }^{18}$ Voir en particulier la clause d'interprétation sur le mariage réservé aux hétérosexuels au début de la loi C-23: « ... the lawful union of one man and one woman to the exclusion of all others ». Voir également l'article 365 C.c.Q., « ... [le mariage] ne peut l'être [être célébré] qu'entre une homme et une femme qui expriment publiquement leur consentement libre et éclairé à cet égard. » 